\title{
Coupling of Er ions to surface plasmons on $\mathrm{Ag}$
}

\author{
J. Kalkman, ${ }^{\text {a) }}$ L. Kuipers, and A. Polman \\ FOM Institute for Atomic and Molecular Physics, Kruislaan 407, 1098 SJ Amsterdam, The Netherlands \\ H. Gersen \\ Applied Optics Group, University of Twente, P.O. Box 217, 7500 AE Enschede, The Netherlands
}

(Received 18 August 2004; accepted 30 November 2004; published online 21 January 2005)

$\mathrm{Er}^{3+}$ ions located $100 \mathrm{~nm}$ beneath the surface of silica glass show an enhanced photoluminescence decay rate when the glass is covered with Ag. Correcting for concentration quenching effects, the decay rate is enhanced by $70 \%$, compared to the case without Ag. The data are in agreement with a model that takes into account variations in local density of states and excitation of surface plasmons and lossy surface waves, resulting in direct evidence for the efficient generation of surface plasmons by excited $\mathrm{Er}^{3+}$ ions. Using the model, optimum conditions for coupling to surface plasmons are derived, which can be used to enhance the emission rate and quantum efficiency of a wide range of Er-doped materials. (C) 2005 American Institute of Physics.

[DOI: $10.1063 / 1.1856133$ ]

Trivalent erbium ions, with their characteristic ${ }^{4} I_{13 / 2}$ $\rightarrow{ }^{4} I_{15 / 2}$ intra- $4 f$ transition at $1.53 \mu \mathrm{m}$, play a key role in a wide variety of optical and optoelectronic devices. They provide the gain medium in optical telecommunication amplifiers and lasers, and provide a source of electroluminescence in a range of Si-based optoelectronic devices. However, due to the parity forbidden character of the intra- $4 f$ transition, the radiative emission rate of $\mathrm{Er}^{3+}$ is very low, typically in the range $100-1000 \mathrm{~s}^{-1}$. Consequently, the output power and modulation speed of Er-doped light-emitting diodes or lasers is limited. Moreover, the low radiative rate makes the Er emission sensitive to nonradiative decay processes, such as Auger recombination ${ }^{1}$ and energy backtransfer in $\mathrm{Si}^{2},{ }^{2}$ or upconversion and concentration quenching in silica glass. ${ }^{3} \mathrm{Sev}-$ eral key limitations in Er-based photonic materials technology could be resolved if the radiative emission rate of $\mathrm{Er}$ could be artificially enhanced. We propose to enhance the Er radiative decay rate by coupling to surface plasmons (SPs). Surface plasmons are propagating electromagnetic waves localized at the interface between a metal and a dielectric, coupled to surface charge oscillations. ${ }^{4}$ An electric dipole, positioned close to such an interface, can decay by the excitation of a SP. As was recently demonstrated for $\mathrm{Er}$ and $\mathrm{Eu}$ ions, the propagating SP can be coupled out into the far field using a grating that relaxes the momentum mismatch between SP and photon. ${ }^{5,6}$ This process thus contributes to the far-field emission of the emitter, effectively increasing its radiative emission rate.

In this letter, we focus on the details of the coupling of an excited Er ion to a metal. We experimentally demonstrate that the photoluminescence decay rate of Er ions in silica glass is enhanced near a $\mathrm{Ag}$ interface. The increase of the decay rate (corrected for concentration quenching effects) is consistent with a model that describes the combined effect of variations in local density of states and excitation of SPs and lossy surface waves. The model is then used to predict optimum conditions for radiative rate and quantum efficiency (QE) enhancements for infrared emission of Er.

The surface region of sodalime-silicate glass samples was doped with $\mathrm{Er}$ by $500 \mathrm{keV} \mathrm{Er}^{+}$ion implantation. Ion

\footnotetext{
${ }^{a)}$ Electronic mail: j.kalkman@amolf.nl
}

fluences ranged from ( 1 to 6$) \times 10^{15} \mathrm{Er} / \mathrm{cm}^{2}$, resulting in peak concentrations ranging from 0.12 to 0.71 at. \%. To avoid charging during the ion implantation the samples were covered with a 40-nm-thick Al layer, which was removed after ion implantation with a $\mathrm{KOH}$ etch. The samples were annealed in vacuum for $1 \mathrm{~h}$ at $512{ }^{\circ} \mathrm{C}$, a procedure known to remove all implantation related defects. ${ }^{3}$ The Er depth profile as measured by Rutherford backscattering spectrometry is described by a Gaussian distribution that is centered at $103 \mathrm{~nm}$ with a straggle of $\sigma=43 \mathrm{~nm}$. Finally, one section of each sample was covered with an optically thick (225 nm) $\mathrm{Ag}$ film using sputter deposition. The dielectric constant of the $\mathrm{Ag}$ film, measured at $\lambda=1538 \mathrm{~nm}$ using ellipsometry, was $\varepsilon_{\mathrm{Ag}}=-41.8+i 4.6$.

Photoluminescence (PL) measurements were performed at room temperature on either $\mathrm{Ag}$ or air covered sections of the Er-implanted samples. An argon-ion laser operating at $488 \mathrm{~nm}$ was used to excite the $\mathrm{Er}^{3+}$ ions into the ${ }^{4} F_{7 / 2}$ manifold. The laser intensity, modulated at $6 \mathrm{~Hz}$ with an acoustooptical modulator, was incident though the transparent back side of the sample. PL was collected from the back using an $f=10 \mathrm{~cm}$ lens and dispersed using a $480 \mathrm{~mm}$ focal-length monochromator, set to a resolution of $6 \mathrm{~nm}$. The collected and dispersed PL intensity was recorded by a liquid-nitrogen cooled Ge diode with a response time of $30 \mu$ s. PL decay traces were taken using a digitizing oscilloscope, integrating a large number or decays.

The inset of Fig. 1 shows a PL spectrum for Erimplanted sodalime-silicate glass covered with Ag. An identical spectrum is observed for the section of the sample without $\mathrm{Ag}$, indicating that the presence of the metal does not affect the crystal field around the $\mathrm{Er}^{3+}$ ions and the atomic dipole transition matrix element is identical for the two cases. Two PL decay traces are shown, taken at the Er peak emission wavelength of $1538 \mathrm{~nm}$, for the sample implanted with $1 \times 10^{15} \mathrm{Er} / \mathrm{cm}^{2}$, for the air- and Ag-covered sections of the sample. The decays are single-exponential, and the PL decay rate for the $\mathrm{Ag}$-covered section $\left(131 \mathrm{~s}^{-1}\right)$ is enhanced compared to the PL decay rate for the air-covered section $\left(87 \mathrm{~s}^{-1}\right)$.

Figure 2 shows the PL decay rates measured at $1538 \mathrm{~nm}$ for the Ag-and air-covered sections of samples with different 


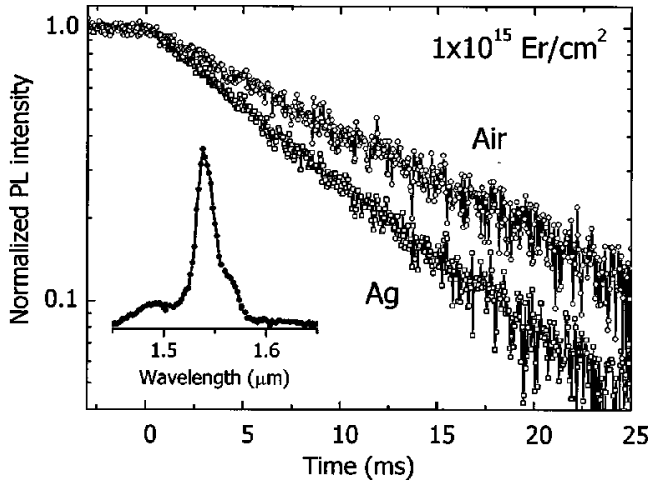

FIG. 1. PL decay curves measured at $\lambda=1538 \mathrm{~nm}$ for a sample doped with $1 \times 10^{15} \mathrm{Er} / \mathrm{cm}^{2}$. The $488 \mathrm{~nm}$ laser was switched off at $t=0$. The decay for the sample in air is slower than that of the sample covered with Ag. The inset shows the Er emission spectrum for the sample covered with Ag.

Er content. The PL decay rate increases with increasing Er concentration, a well-known effect that is attributed to concentration quenching (a process due to Förster energy transfer between Er ions, followed by quenching). ${ }^{3}$ Our previous work has shown that the main quencher in these glasses are $\mathrm{OH}$ impurities, ${ }^{3}$ whose first stretch-vibration overtone is resonant with the $1.5 \mu \mathrm{m}$ transition of $\mathrm{Er}^{3+}$. If the concentration of quenchers is much smaller than the concentration of $\mathrm{Er}^{3+}$ ions, as is the case for our samples, the PL decay rate is described by 8,9

$$
W_{\mathrm{PL}}=W_{\text {int }}+4 \pi C_{\mathrm{Er}-\mathrm{Er}} N_{Q} N_{\mathrm{Er}}
$$

with $W_{\text {int }}$ the $\mathrm{Er}^{3+}$ intrinsic decay rate in absence of concentration quenching, $C_{\mathrm{Er}-\mathrm{Er}}$ an Er-Er interaction constant, $N_{Q}$ the concentration of quenchers, and $N_{\mathrm{Er}}$ the $\mathrm{Er}^{3+}$ concentration. Fitting Eq. (1) to the two data sets in Fig. 2 we find the extrapolated $\mathrm{Er}$ decay rate in absence of concentration quenching: $W_{\text {int,air }}=54 \pm 7 \mathrm{~s}^{-1}$ for the air section and $W_{\text {int, Ag }}$ $=92 \pm 4 \mathrm{~s}^{-1}$ for the Ag section. Adding the Ag film onto the glass thus enhances the intrinsic Er decay rate by $70 \pm 25 \%$. From the slopes in Fig. 2, and using an $\mathrm{OH}$ (quencher) concentration of $8 \times 10^{18} \mathrm{OH} / \mathrm{cm}^{3}$ (Ref. 3) we find $C_{\mathrm{Er}-\mathrm{Er}}$ $=(2.7-3.1) \times 10^{-39} \mathrm{~cm}^{6} / \mathrm{s}$, which is a typical value for sodalime silicate glass. ${ }^{3}$

We describe the interaction between an oscillating dipole and a metal interface by a model developed by Ford and Weber. ${ }^{10}$ In this one-dimensional model, a harmonic oscilla-

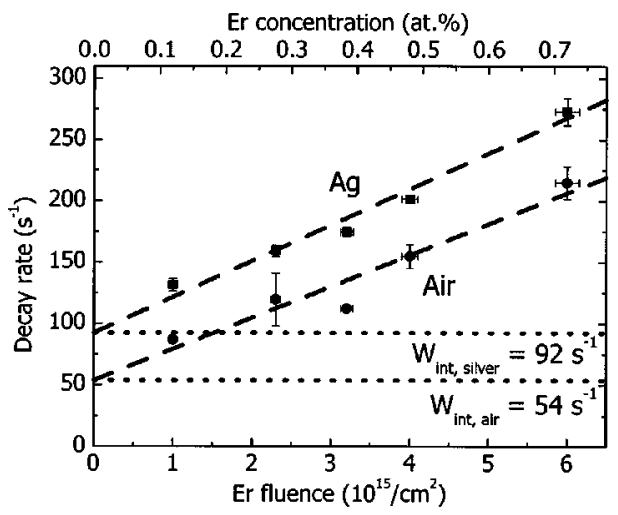

FIG. 2. PL decay rates measured at $\lambda=1538 \mathrm{~nm}$ for samples implanted with different Er fluences, for the sample in air or covered with Ag. The decay rate increases linearly with concentration due to concentration quenching. The dotted horizontal lines indicate the extrapolated decay rates at zero Er

concentration.
Downloaded 24 Jan 2005 to 192.87.153.106. Redistribution subject to AIP license or copyright, see http://apl.aip.org/apl/copyright.jsp tor dipole is driven at constant power and placed in a dielectric layer, which is embedded in a stack of layers with arbitrary dielectric constants. The power dissipation of the dipole is determined by calculating the imaginary part of the electric field at the position of the dipole. The dipole moment times the electric field is due to contributions from the dipole, and the fields reflected by the dielectric surrounding the dipole. After a spatial Fourier transform of the dipolar field into plane waves, the reflected fields can be calculated using the Fresnel reflection coefficients for all interfaces. The total power dissipation is then calculated for all in-plane wave vectors $\left(k_{\|}\right)$. If the dipole is embedded in an infinitely thick dielectric layer, power is dissipated only for in-plane wave vectors that are smaller than the dipole's far-field wave vector in its embedding dielectric medium $\left(k_{\|} \leqslant k_{d}=(\omega / c) \sqrt{\varepsilon_{d}}\right)$, i.e., evanescent waves do not transport energy. However, if an absorbing medium with a smaller real part of the dielectric constant is located within the near-field of the dipole, power is also dissipated for wave vectors $k_{\|}>k_{d}$. Using Ford and Weber's model, we calculated the power dissipation of a dipole emitting at $\lambda_{\mathrm{vac}}=1538 \mathrm{~nm}$ (the $\mathrm{Er}^{3+}$ peak emission wavelength) in sodalime silicate glass $\left(\varepsilon_{d}=2.25\right)$ both for an air- $\left(\varepsilon_{\text {air }}=1\right)$ and Ag-covered section $\left(\varepsilon_{\mathrm{Ag}}=-41.8+i 4.6\right)$.

Figure 3(a) shows the power dissipation spectrum at $\lambda_{\mathrm{vac}}=1538 \mathrm{~nm}$ calculated for a randomly oriented dipole at a distance $d=103 \mathrm{~nm}$ (the peak depth of the Er distribution in the experiments) for the case of the glass in air. As can be seen, power is dissipated for wave vectors $k_{\|} / k_{d} \leqslant 1$. The cut-off at $k_{\|} / k_{d}=1$ (dashed vertical line) shows that all power is dissipated into the far field, and that there is no absorption for larger wave vectors. Figure 3(b) shows the dissipated power spectrum for the corresponding case for the dielectric covered with Ag. The power dissipation shows the spontaneous emission contribution for $k_{\|} / k_{d} \leqslant 1$. A large peak is observed at $k_{\|} / k_{d}=1.027$ (see the inset), which corresponds to the wave vector of a SP at the $\mathrm{Ag} /$ silica glass interface. Power dissipation for even larger wave vectors is due to the excitation of lossy surface waves: energy transfer to the metal owing to the imaginary part of the dielectric constant (i.e., electron scattering). ${ }^{10,11}$

The total decay rate is calculated by dividing the power dissipation, integrated over the complete wave vector range, by the energy of the dipole field. The decay rates are averaged over parallel and perpendicular orientations relative to the interface, and normalized relative to the bulk value. Calculations were performed for distances from the interface in the range of $1-1500 \mathrm{~nm}$, and convergence to within $1 \%$ was achieved for all distances larger than $5 \mathrm{~nm}$. To partition the total decay rate of the dipole ions near the Ag/glass interface into the three decay channels, the spectra in Fig. 3(b) were integrated over the corresponding wave vector ranges (spontaneous emission: $k_{\|} / k_{d}<1$, SP excitation $1 \leqslant k_{\|} / k_{d}<1.056$, lossy surface waves: $\left.1.056 \leqslant k_{\|} / k_{d} \leqslant 200\right)$.

Figure 3(c) shows the normalized spontaneous emission rate (equal to the total decay rate) of the dipole for the case of the dielectric in air, as a function of distance from the surface. The decay rate shows an oscillation with depth due to the reflection of emitted waves that, by either constructive or destructive interference, decreases or increases the dipoles decay rate. The oscillations represent variations in the local density of states, an effect that previously has been probed experimentally by placing Er or Eu ions at different distances from the interface. ${ }^{12-14}$ The reduced decay rate near the in- 

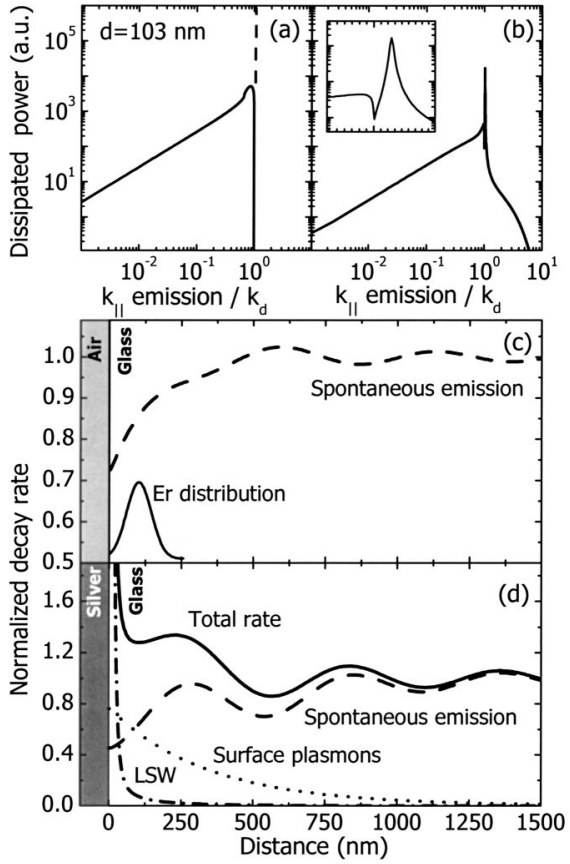

FIG. 3. Power dissipation as a function of normalized in-plane wave vector, calculated for a randomly oriented dipole emitting at $\lambda_{\mathrm{vac}}=1538 \mathrm{~nm}$ and positioned at $d=103 \mathrm{~nm}$ for (a) an air/glass interface, and (b) an Ag/glass interface. The large peak in (b) is due to coupling to surface plasmons, a feature that is more clearly illustrated in the inset. Calculated normalized decay rate as a function of distance from (c) the air/glass interface, and (d) the $\mathrm{Ag} / \mathrm{glass}$ interface. For (d) the total decay rate is split up into three decay channels, including lossy surface wave excitation (LSW).

terface is a result of the requirement of continuity of $\boldsymbol{D}$ fields parallel to the interface.

Figure 3(d) shows the depth-dependent normalized decay rates for the glass covered with Ag. The spontaneous emission rate shows oscillatory behavior as in Fig. 3(c), though with a larger decrease near the surface, due to the higher reflectivity of the interface. Figure 3(d) also shows a clear SP excitation component that exponentially decreases with depth as the dipolar near field moves further away from the $\mathrm{Ag}$. The figure shows that for very small distances $(d$ $<20 \mathrm{~nm}$ ) the decay is dominated by the generation of lossy surface waves. At $d=90 \mathrm{~nm}$ the SP excitation rate is roughly equal to the spontaneous emission rate.

The effect of the various decay processes on the experimentally prepared Er-doped samples can be evaluated by integrating the depth-dependent rates for each of the decay channels over the Er depth distribution [shown in Fig. 3(c)]. From this analysis it follows that the total decay rate on the $\mathrm{Ag} / \mathrm{glass}$ interface is enhanced by a factor of 1.52 , relative to the total decay rate for the air/glass interface. This is close to the experimentally determined ratio of intrinsic decay rates of $1.7 \pm 0.25$ derived from Fig. 2 .

The correlation between experiment and theory presented here demonstrates that $\mathrm{Er}^{3+}$ ions can couple efficiently to $\mathrm{SPs}$ at the $\mathrm{Ag} /$ silica glass interface. This provides direct support for our earlier study ${ }^{6}$ that showed large modifications in the spontaneous emission spectrum of Er ions embedded in a metallodielectric grating that were interpreted in terms of the near-field generation of SPs by excited $\mathrm{Er}^{3+}$ ions. To illustrate how coupling to SPs may eventually serve to enhance the Er emission QE we evaluate data for the sample with the highest Er concentration in Fig. 2. The Er ions for the air-covered sample have an internal QE of 26\%, due to concentration quenching. The Er ions in the sample covered with $\mathrm{Ag}$ show a further enhanced decay rate due to coupling to SPs. If the latter can be coupled out into the far field at an efficiency of $100 \%$, the external QE would increase to $35 \%$. Note that extraction efficiencies for SPs as high as $80 \%$ have already been demonstrated. ${ }^{15}$

Although the emission rate and QE enhancements presented here are relatively small, they clearly demonstrate the concept of coupling to SPs in the near-infrared. As the experiments are in agreement with theory, we can now use the latter to search for more effective ways to enhance emission rates and QEs by coupling to SPs. The relatively weak coupling of Er to SPs at the Ag/silica glass interface is caused by the large difference between the Er emission frequency and the Ag plasma frequency. ${ }^{15}$ There are several ways to increase the coupling to SPs (and thereby the decay rate and QE enhancement): (1) using a dielectric with higher dielectric constant (e.g., Si), (2) using a different metal (e.g., Au), or (3) by coupling to a thin metal film rather than an optically thick layer. ${ }^{14}$ Calculations based on the previously described model show that the SP excitation rate for a dipole in Si $\left(\varepsilon_{d}=11.9\right)$ emitting at $\lambda_{\mathrm{vac}}=1538 \mathrm{~nm}$ and positioned at a distance of $20 \mathrm{~nm}$ from a $10 \mathrm{~nm}$ thin $\mathrm{Ag}$ film is 12 times faster than the bulk spontaneous emission rate.

In conclusion, the decay rate of optically excited $\mathrm{Er}^{3+}$ ions near a glass surface is enhanced by $70 \%$ if the glass is covered with Ag. The enhancement is in good agreement with a model that takes into account variations in the local density of states, excitation of surface plasmons and lossy surface waves, with the majority of the decay rate enhancement due to SP excitation. Much larger enhancements are expected for an optimized metallodielectric geometry. If these SPs can be efficiently coupled out, the emission rate and $\mathrm{QE}$ of Er can be enhanced. In this way, fundamental quenching processes of $\mathrm{Er}$ in $\mathrm{Si}$ and silica glass may be avoided. The next challenge is to experimentally demonstrate this.

The authors acknowledge M. J. A. de Dood for fruitful discussions and help in sample preparation. This work is part of the research program of the "Stichting voor Fundamenteel Onderzoek der Materie (FOM)," which is financially supported by the "Nederlandse organisatie voor Wetenschappelijk Onderzoek (NWO)."

${ }^{1}$ F. Priolo, G. Franzò, S. Coffa, and A. Carnera, Phys. Rev. B 57, 4443 (1998).

${ }^{2}$ N. Hamelin, P. G. Kik, J. F. Suyver, K. Kikoin, A. Polman, A. Schönecker, and F. W. Saris, J. Appl. Phys. 88, 5381 (2000).

${ }^{3}$ A. Polman, J. Appl. Phys. 82, 1 (1997).

${ }^{4}$ H. Raether, Surface Plasmons (Springer, Berlin, 1988).

${ }^{5}$ P. Andrew and W. L. Barnes, Phys. Rev. B 64, 125405 (2001).

${ }^{6}$ J. Kalkman, C. Strohhöfer, B. Gralak, and A. Polman, Appl. Phys. Lett. 83, 30 (2003).

${ }^{7}$ P. G. de Gennes, J. Phys. Chem. Solids 7, 345 (1958).

${ }^{8}$ M. J. Weber, Phys. Rev. B 4, 2932 (1971).

${ }^{9}$ G. W. Ford and W. H. Weber, Phys. Rep. 113, 195 (1984).

${ }^{10}$ W. L. Barnes, J. Mod. Opt. 45, 661 (1998).

${ }^{11}$ K. H. Drexhage, J. Lumin. 1-2, 693 (1970).

${ }^{12}$ E. Snoeks, A. Lagendijk, and A. Polman, Phys. Rev. Lett. 74, 2459 (1995).

${ }^{13}$ R. M. Amos and W. L. Barnes, Phys. Rev. B 55, 7249 (1997).

${ }^{14}$ J. Moreland, A. Adams, and P. K. Hansma, Phys. Rev. B 25, 2297 (1982).

${ }^{15}$ H. Morawitz and M. R. Philpott, Phys. Rev. B 10, 4863 (1974). 